Itinéraires Itinéraires

Littérature, textes, cultures

2018-2 et $3 \mid 2019$

Les imaginaires de la traduction

\title{
Avec qui traduit-on? Les imaginaires de la traduction poétique
}

With Whom do We Translate? The Imaginaries of Poetry Translation

\section{Christine Lombez}

\section{OpenEdition}

Journals

Édition électronique

URL : http://journals.openedition.org/itineraires/4561

DOI : $10.4000 /$ itineraires.4561

ISSN : 2427-920X

Éditeur

Pléiade

Référence électronique

Christine Lombez, «Avec qui traduit-on ? Les imaginaires de la traduction poétique », Itinéraires [En ligne], 2018-2 et 3 | 2019, mis en ligne le 20 février 2019, consulté le 18 juin 2019. URL : http:// journals.openedition.org/itineraires/4561 ; DOI : 10.4000/itineraires.4561

Ce document a été généré automatiquement le 18 juin 2019.

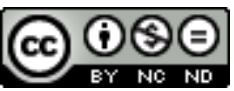

Itinéraires est mis à disposition selon les termes de la licence Creative Commons Attribution - Pas d'Utilisation Commerciale - Pas de Modification 4.0 International. 


\title{
Avec qui traduit-on? Les imaginaires de la traduction poétique
}

\author{
With Whom do We Translate? The Imaginaries of Poetry Translation
}

\author{
Christine Lombez
}

1 Nul n'est seul devant la page blanche, qu'il soit auteur ou traducteur, si l'on en croit les propos du poète palestinien Mahmoud Darwich (2006:31) : « la page blanche est en fait déjà noircie par d'autres écritures. Selon T. S. Eliot, être mûr sur le plan littéraire, c'est se rappeler nos ancêtres, c'est sentir qu'il y a derrière chaque texte poétique toute une lignée d'ancêtres ». Loin d'être un obstacle à la création, la reconnaissance de cette «mémoire» serait même le signe d'une destinée poétique arrivée à son point de maturité...

2 Si l'on considère que traduire est pleinement un acte d'écriture, avec qui le traducteur de poésie traduit-il ? Dans quelle mesure est-il accompagné dans sa tâche par d'autres voix, d'autres imaginaires que les siens, mais qui vont donner à sa création toute son épaisseur et sa saveur littéraires? Il y a là un phénomène de dialogisme - voire d'intertextualité - d'autant plus intéressant à questionner qu'il informe souvent les choix interprétatifs du traducteur, surtout quand celui-ci est poète lui-même. On se demandera en quoi le texte-traduction ainsi produit peut se lire comme un feuilleté d'imaginaires poétiques, quel rapport (empathique ou antagoniste) ces derniers entretiennent avec l'original étranger et dans quelle mesure ils sont susceptibles d'en renouveler, in fine, la lecture.

\section{Traduction et imaginaire(s) : noms et paratextes}

De nombreuses images et métaphores ont désigné en Europe, le fait est connu, la traduction au cours des âges et dans les langues les plus diverses: il y est souvent question de voyage, de traversée, de transformation ou de conversion d'une réalité 
première dans une autre. Incontestablement, le mot traduire trahit son origine latine ( traducere), même si, à Rome, jamais ce verbe au périmètre sémantique surtout spatial (on en a conservé des traces en français avec une expression comme "traduire en justice ») n'a eu le sens dérivé qu'on lui connaît aujourd'hui' ${ }^{1}$. On comprend ainsi que l'imaginaire occidental de la traduction comme déplacement de sens, passage ou voyage s'en soit trouvé affecté et que des cultures non européennes aient pu avoir de tout autres représentations sur le sujet ; c'est notamment le cas en Chine ancienne où, comme le fait remarquer Bellos (2012 : 40-41),

le titre qui vous désignait si vous occupiez la fonction de traducteur officiel dépendait de la frontière impériale à laquelle vous étiez affecté. [...] Jamais personne n'a songé à expliquer la traduction en termes de « transport du sens d'une langue dans une autre ».

4 Au-delà du mot qui le désigne, un imaginaire propre à chaque langue est également à l'œuvre dans le processus traductif. Il permet en effet d'apercevoir des particularités qui font le fameux " génie des langues » et compliquent la tâche des traducteurs au point où l'on peut parfois parler d'intraduisibilité. Ainsi, il n'est pas indifférent de constater que la mort a un genre différent selon qu'il s'agit de langues romanes (où elle est féminine) ou germaniques (elle y est vue comme une entité masculine). L'érotisme du baiser de la mort à la jeune fille se voit donc perdu dans une langue romane comme le français (qui met en présence deux femmes), tandis qu'il reste bien présent en allemand (on pensera au Lied de Schubert «Der Tod und das Mädchen » qui traduit subtilement ce jeu de séduction homme / femme). Dans un autre contexte, cité par R. Jakobson, le fait que le mot vie soit féminin en russe est des plus signifiants pour le recueil de Boris Pasternak intitulé justement Ma scur la vie. Que faire quand on doit le traduire dans une langue comme le tchèque (ou le serbe) où vie est du genre masculin? Devra-t-on aller jusqu'à changer le référent et à parler de "frère »? La traduction serbe a tranché en faisant cohabiter les deux genres (féminin pour sœur, masculin pour vie). La question se pose également pour les astres, souvent présents en poésie : l'étoile ou la Lune ${ }^{2}$ sont féminines en français (et dans bon nombre de langues dérivées du latin), masculines dans les langues germaniques. L'effet produit dans la langue d'arrivée et l'imaginaire créé seront à chaque fois bien différents à la suite des choix effectués par le traducteur. Cela est très visible dans le poème de Neruda «Los Verdugos » issu du Canto General, dans la scène relatée au chant v, où, écrit le poète, "el gato y la escorpiona fornicaron / en la patria selvática ». Si les protagonistes sont mâle et femelle en espagnol, en français aussi bien le chat que le scorpion sont du genre masculin... Le traducteur n'a donc qu'une alternative : soit (cas d'Alice Ahrweiler en 1952) traduire «le chat et le scorpion ont forniqué / dans la patrie sauvage » (scène susceptible d'engendrer une certaine perplexité du côté du lecteur), soit changer l'un des référents, afin de conserver le couple générique initial. C'est le choix fait par Claude Couffon, ami de Neruda retraduisant l'œuvre en 1977, avec « le chat a forniqué avec la tarentule / dans notre patrie forestière ». Ce qui, en dépit de la fidélité au genre grammatical des protagonistes dans l'original, ne suffit pas à dissiper tout étonnement devant l'étrangeté de l'action, mais permet au moins de conserver le couple masculin / féminin imaginé par le poète...

5 Les catégories propres à la langue conditionnent donc dans une certaine mesure les représentations qu'elle produit, ce qui les rend non superposables d'un idiome à l'autre. On souscrira ainsi aux propos de Cary pour qui la traduction littéraire n'est pas une opération seulement «linguistique». Tout ne peut se décrire, en effet, en termes linguistiques lors de la traduction littéraire: si la langue est porteuse d'obstacles qui lui 
sont propres (et qui relèvent en effet de la linguistique), la traduction met en jeu une démarche plus complexe où ce sont en fait des réalités impalpables, fruits de l'imaginaire de l'auteur et de son traducteur, qui se font face (la fameuse "pesée» des mots de Larbaud) et donnent toute son épaisseur au texte traduit.

Les préfaces et autres discours d'accompagnement (notes, postfaces) permettent de mieux cerner l'imaginaire $\mathrm{du}$ traducteur qui y exprime souvent une vision très personnelle rendant compte de ses choix. On citera ici trois exemples représentatifs des conséquences que peut faire peser l'imaginaire d'un traducteur sur son texte.

7 Traduisant à titre « d'amusement» le début de l'Iliade d'Homère durant la guerre ${ }^{3}$, le poète-traducteur et ami d'Apollinaire Pierre Albert-Birot (PAB) tient dans son discours liminaire des propos très révélateurs sur l'importance que les représentations subjectives que l'on peut avoir sur un mot ( $a$ priori assez banal) est ensuite susceptible d'engendrer à la lecture puis à la traduction :

Il s'agit du mot « k $\lambda$ เolov» que tous les traducteurs ont traduit par «tente », on parle très souvent de la tente d'Achille. Or le professeur Paul Mazon a dit: la tente militaire est chose relativement moderne, les Grecs devant Troie n'avaient pas de tentes, et d'autant moins qu'ils étaient «campés » là depuis dix ans, il faut donc prendre ledit mot dans son vrai sens qui est construction provisoire, appentis rudimentaire appuyé au mur d'une maison de maître, voire même de hangar, étable, enfin quelque chose s'apparentant avec ce que la guerre moderne emploie à l'arrière, le baraquement, la BARAQUE. Et dans cette nouvelle traduction, Achille n'entre plus sous sa tente, mais dans sa baraque. Comme bien on pense, à cette nouvelle j'ai ouvert l'œil, moi qui avais accepté sans discussion le mot «tente », et en réalité, c'est bien vrai, il ne l'a peut-être qu'à force de bonne volonté de la part des auteurs de dictionnaires, et j'étais tout près de venir, moi aussi, après $\mathrm{M}$. Paul Mazon, à quelque hangar ou baraquement, ce qui d'ailleurs doit être la vérité, on voit très bien, quand on raisonne, ce camp de Grecs comme une sorte de grand village fait d'un fouillis de baraques dressées n'importe comment les unes à côté des autres, mais, mais, mais... quand Agamemnon dit: Allez à la tente d'Achille, immédiatement l'image complète d'un camp de guerriers, avec toute sa vie et son odeur militaires se forme devant nous, tandis que s'il disait: Allez aux baraques d'Achille, aucune image guerrière ne vient devant nous ; or en pareil cas la vertu verbale est tellement à considérer que sans hésiter, j'ai gardé le sens « complaisant » de tente, estimant qu'ici, la vraie vérité est à côté de la vérité.

[...] le mot grec contient bien, je crois, les sens de construction provisoire et de plan incliné, ce qui s'accorde on ne peut mieux avec la tente. En tout cas, au seul mot "tente", nous voyons un champ de guerre, au mot "baraque", nous voyons un vague chantier de grands travaux, ou peut-être, à l'heure actuelle, un camp de prisonniers. Vraiment, vraiment, nous perdons tout, c'est trop. (Mars 1944) (AlbertBirot $1945: 12-13$ )

8 «Tente » ou «baraque »? Il est évident ici que c'est l'imaginaire du traducteur, en lien avec le contexte de la guerre et des camps durant les années 1940, qui l'empêche de choisir « baraque », le terme sans doute le plus exact. En effet, ce mot projette devant ses yeux une image insupportable : « au seul mot "tente", nous voyons un champ de guerre, au mot "baraque", nous voyons un vague chantier de grands travaux, ou peut-être, à l'heure actuelle, un camp de prisonniers. Vraiment, vraiment, nous perdons tout, c'est trop » (Ibid.). PAB choisit donc sciemment, en vertu de la représentation qu'il s'en est fait, un mot plus inexact, car plus neutre, mais qui a pour lui l'avantage de ne pas contrarier sa vision personnelle du camp grec chez Homère (qu'il ne veut en rien voir ressembler à un camp de concentration ou de prisonniers dans la France de l'Occupation). 
Un autre exemple frappant de l'ombre projetée de l'imaginaire d'un traducteur se lit dans la célèbre préface donnée par Armel Guerne, poète-traducteur suisse, à sa traduction de Novalis. En accord avec sa conviction selon laquelle Novalis (à qui il s'est plus ou moins identifié) aurait été " cette âme latine dans son corps allemand et son verbe germain » (1975: 8), Guerne affirme son désir, non de traduire, mais bien de re-penser intégralement une œuvre à ses yeux emprisonnée dans la langue allemande, pour l'en délivrer :

Nécessité spirituelle implicite, non pas (de) la traduction toujours plus ou moins faisable, non pas (de) la naturalisation proprement impensable, mais la re-pensée en français dans tout ce qu'elle peut avoir de légitime, de la pensée de Novalis qui aspire à des gestes, à des mouvements qu'empêtre ou que gêne aux entournures son costume allemand. [...] - une sorte de besoin initial, dont la satisfaction lui donne ou lui « rend » quelque chose, en dépit de tout ce que lui fait perdre au passage, sous la seule responsabilité du re-penseur, la re-pensée, et par la seule faute du traducteur, la traduction. (Guerne $1975: 27$ )

10 Une telle pétition de principe, aspirant à gommer toute la germanité inhérente au texte original, ne pourra que peser sur le texte français (il n'est qu'à lire la violence des propos de Guerne à l'égard de l'un des traducteurs de Novalis, M. de Gandillac, qui lui n'a pas choisi la même voie - «ce que je sais bien, c'est que son plus précieux outil de travail est un sécateur coupeur d'ailes, et après, pour fignoler, le marteau-pilon ${ }^{5} »-$ pour s'en faire une idée).

11 Dernier cas envisagé, les paratextes ${ }^{6} \mathrm{~d}^{\prime}$ Yves Bonnefoy à ses traductions (il en refit certaines) de la poésie de Shakespeare. Si Bonnefoy est connu comme le traducteur enthousiaste du théâtre shakespearien, il est aussi l'auteur d'une version française du poème Vénus et Adonis ainsi que du cycle des Sonnets qu'il aborde avec certaines réserves, allant jusqu'à émettre des doutes sur leur paternité réelle. Pour Bonnefoy en effet, à supposer que Shakespeare soit bien l'auteur des Sonnets, ce n'est pas dans un but de célébration amoureuse mais afin de dénoncer la poésie rhétorique et conceptuelle de l'époque $d^{\prime}$ 'Elizabeth ${ }^{7}$. Cette vision très personnelle des œuvres (dans laquelle on peut voir un reflet des combats que Bonnefoy mène parallèlement dans sa propre écriture, déchirée entre tentation abstraite et désir d'immédiateté) conduira à des choix de traduction qui ne le sont pas moins: choix de la prose pour Vénus et Adonis (alors que d'ordinaire Bonnefoy est un fervent partisan de la traduction en vers), « agrandissement » de certains sonnets par l'ajout de vers surnuméraires, qui échappent ainsi à leur forme classique contrainte $(16,17$ et même 20 , au lieu des 14 vers groupés en trois quatrains et un distique). Dans les deux cas, il s'agit pour lui d'en restituer tout l'aspect rhétorique et métapoétique dénonçant les travers de la « poésie close » (conceptuelle et hermétique) au profit de la "poésie ouverte» (le théâtre) (Bonnefoy 2013a: 71-72). L'imaginaire d'un traducteur se révèle donc une matrice de représentations prégnante qui laisse dans sa traduction des traces formelles très concrètes, d'autant plus s'il rentre en conflit avec les instructions données par le texte étranger.

\section{Conflits d'énonciations et intertextualités agonistiques}

12 Avec qui (contre qui) traduit-on ? La notion de « lecture-écriture » forgée par Meschonnic (1972 : 50) pose à l'évidence la question de la subjectivité du traducteur et de la marge de manœuvre dont celui-ci dispose lorsqu'il traduit un texte littéraire. Nul ne procède en effet jamais ex nihilo: il y a toujours chez qui traduit une mémoire implicite nourrie de lectures passées pouvant donner lieu à interférences, de vécu personnel, d'expériences 
diverses, qui conditionnent le rapport au texte étranger. Certaines traductions s'effectuent en outre dans un rapport à l'original déjà particulier voire problématique, telles celles d'Edgar Allan Poe par Charles Baudelaire qui voyait dans l'écrivain américain un véritable alter ego, le cas déjà cité de Guerne et de Novalis, ou encore Marina Tsvetaeva traduisant celui qu'elle nomme «son » Pouchkine. Bonnefoy n'a pas manqué d'évoquer la "mémoire poétique» comme paramètre à intégrer selon lui à la lecture de toute traduction, allant même jusqu'à théoriser une pratique singulière de la traduction, «la traduction au sens large ». Dans son analyse comparée du «Corbeau » de Poe traduit par Baudelaire et Mallarmé (2013:67, 70, 74-76), il affirme que la véritable traduction du poème serait, à ses yeux, davantage à chercher dans leur œuvre personnelle (et, en l'espèce, dans « La chambre double » et le « Sonnet en yx ») :

Ne devrait-on pas les considérer plutôt comme des traductions véritables de celuici, et même les vraies traductions... ? [...] J'appelle traduction au sens large ces réactions du traducteur qui s'ajoutent à sa traduction au sens habituel et étroit du mot : qui s'y ajoutent ou même s'y substituent. Et je pense qu'il y a sens à considérer ces événements de la profondeur [...], s'attacher à ce second cercle du traduire. (Bonnefoy 2013b : 75)

13 Dans le cas d'un poète traducteur, l'œuvre tout entière qu'il a produite devra ainsi être convoquée pour la lecture de ses traductions dans la mesure où « [...] ce que le traducteur n'aura pu garder de l'œuvre qu'il a traduite se sera reformé dans son œuvre à lui à même niveau de conscience poétique [...]» (Bonnefoy 2013b : 82). La correspondance ne se fera plus donc de texte source à texte cible, mais bien en "écoute par œuvres interposées " (26), du texte original à l'œuvre globale du traducteur, l'imaginaire (ce qu'il nomme les «événements de la profondeur ») qui informe celle-ci expliquant / reflétant le processus ayant conduit au texte cible.

14 «Ces réactions du traducteur qui s'ajoutent à sa traduction » créent de facto un espace polyphonique pouvant parfois mener à des conflits d'énonciations. On trouve des exemples particulièrement éclairants de ces subtiles "transactions» chez les poètes traducteurs. Armand Robin, par exemple, conçoit la traduction comme la projection d'une subjectivité (la sienne) dans celle de l'auteur étranger, au point que sa voix (à lui Robin) devient plus audible que celle du poète original : «D'autre en autre je foisonne!", affirme-t-il avec panache (1992: 49), résumant ainsi sa carrière de "non-traducteur ». C'est également vrai, alternativement, pour Saint John Perse et T. S. Eliot ${ }^{8}$. Quand, dans le poème «We are the Hollow Men », Perse rend l'épigraphe "A penny for the Old Guy " (cri des enfants lorsqu'ils demandent de l'argent pour acheter de quoi faire un feu d'artifice lors du Guy Fawkes Day) par "Aumône aux hommes de peu de poids", le rapport avec le contexte d'énonciation chez Eliot est bien moindre qu'avec l'imaginaire poétique de Saint John Perse. De même, l'univers propre à S. Beckett se reflète très fortement dans ses traductions d'Apollinaire (le poème "Zone » en est un bon exemple, notamment quand il traduit la périphrase « maladie honteuse » par « syphilis », mot que le poète français n'avait pas osé écrire dans son poème en 1913, mais tout à fait cohérent dans le cosmos et l'esprit beckettiens...).

Cette superposition peut revêtir des aspects extrêmes, comme dans les traductions de Paul Celan par André Du Bouchet-une réalité dénoncée avec virulence par Henri Meschonnic dans une célèbre controverse ("On appelle cela traduire Celan») où il va jusqu'à parler de «massacre» (1973: 369). Dans le paragraphe intitulé «Celan en Du Bouchet ", Meschonnic passe en revue les éléments qui lui permettent de conclure que chez le poète traducteur français, «traduire un poème est en retrait sur écrire. Ce qui 
affleurait rarement dans ses poèmes, qui sont plus dominés, se déverse dans son traduire » (ibid. : 388). On ne saurait mieux dire à quel point il s'agit ici d'une forme de «traduction-annexion» effectuée par l'instance traduisante en accord avec son imaginaire poétique personnel (est-ce une coïncidence? Celan avait fait part de sa réticence à être traduit en français ${ }^{9}$ ). Les exemples cités par Meschonnic étant devenus célèbres, on n'en citera qu'un parmi les plus significatifs : « zwei/Mundvoll Schweigen » (litt. « deux/bouchées de silence ») rendu par Du Bouchet en « deux bouches qu'a silence saturées ». La traduction s'apparente dès lors à un palimpseste, un texte multiple qui conserve, pour ainsi dire, la « mémoire poétique » de deux imaginaires personnels.

Les interférences perceptibles dans le cas de traductions d'écrivains nous rapprochent de réels phénomènes d'intertextualité, des échos de l'univers personnel du traducteur créant un brouillage (Riffaterre parlerait d'« agrammaticalité ») dans le texte-traduction. Mais l'intertextualité, consciente ou non, révélatrice d'un imaginaire singulier, est parfois déjà présente chez l'auteur étranger; elle pourra être identifiée ou non, acceptée ou non, par le traducteur. Dans tous les cas, les conséquences sur la lecture du texte original seront fortes. Chez Darwich, la citation (déclarée) de deux vers de Yeats dans le poème «Lorsqu'il s'éloigne" (2006: 365) est tout sauf innocente : là où le texte de Yeats («An Irish airman foresees his death", 1919) portait «Those that I fight I do not hate / Those that I guard I do not love » (litt. «Je ne hais pas ceux que je combats / Je n'aime pas ceux que je garde »), Darwich écrit « Je n'aime pas ceux que je défends / Tout comme je n'ai pas d'adversité contre ceux que je combats ", introduisant, par l'inversion des propositions, une nuance d'autant plus subtile que le locuteur est un officier israélien. Le traducteur, alerté par le renvoi de Darwich, devra bien se garder de rétablir l'ordre initial présent chez Yeats. Autre cas: l'hypotexte dantesque chez Beckett (visible à des allusions fugitives à des personnages comme Bocca, Malacoda, etc.) ou chez T. S. Eliot dans The Waste Land sera à chaque fois à prendre en considération dans tout projet de traduction. On a déjà évoqué ailleurs (Lombez 2016: 157 et suiv.) le cas de Georg Trakl et la nécessité de traduire selon Meschonnic, dans le poème « Die schöne Stadt» («La Belle Ville»), le mot Tor par "porte» et non "portail» en raison du lien intertextuel existant avec un texte de Maeterlinck, «Les sept filles d'Orlamonde». De même, il est évident qu'un traducteur étranger ayant à s'occuper de l'œuvre d'un poète comme Philippe Jaccottet devra rendre perceptible les liens (thématiques et stylistiques) que celui-ci entretint, au moins à ses débuts, avec la poésie de Rilke, qui imprègne son écriture (notamment dans Requiem et L'Effraie). Quant au roman Malina d'Ingeborg Bachmann, par exemple, il est truffé de citations non déclarées du poète allemand Paul Celan avec qui elle vécut un temps une relation passionnée. Partie intégrante de l'œuvre de Bachmann, la strate poétique celanienne devrait non seulement être identifiée à la lecture mais aussi rendue par le traducteur dans sa langue comme vecteur herméneutique signifiant.

Cependant, l'épaisseur intertextuelle des textes comme indice d'un imaginaire littéraire à l'œuvre pose souvent des problèmes à la traduction car s'il s'agit, dans un premier temps, de repérer le phénomène dans l'original (ce qui n'est pas toujours aisé en l'absence de notes pour les renvois les plus discrets), il faudra ensuite recréer dans la traduction un équivalent pertinent, et, dans le meilleur des cas, parlant à l'imaginaire du lecteur de la langue cible. Jörn Albrecht (1998: 247-248) mentionne le cas singulier de "Téhéran 68 ", poème en prose d'Alain Lance à forte connotation politique, et sa traduction en allemand du temps de la RDA par le poète Völker Braun. Sur fond de vie quotidienne dans la capitale iranienne, partagée à la fin des années 1960 entre tradition et modernité, Lance 
décrit l'emprise de l'armée et de la police du Shah, terminant son texte par une citation ironiquement détournée d'un vers de Verlaine: «Et voici des cars des casques et des crosses $^{10}$ ». Ce renvoi intertextuel subtil (y compris sur le plan des sonorités, elles-mêmes subverties) trouvera cependant sa traduction dans la version allemande grâce à la sagacité de Braun: après une première version encore assez proche d'un mot à mot ("Und hier sind Karren Ketten Kolben»), le traducteur se risque à un autre choix, également très ironique dans le contexte : il reprend en le détournant un vers de Goethe extrait de "Mailied ", poème d'amour largement connu, et remplace le référent bucolique (Natur) par une réalité militaire (Montur) ce qui, dans le contexte ne manque pas de sel ${ }^{11}$. Il y a également le cas des citations métriques qui, si elles sont repérées, vont solliciter tous les ressorts de la créativité car elles ne sont pas dépourvues d'intentions - souvent ironiques. André Markowicz relève ainsi le cas des citations de mètres (antiques mais pas seulement ${ }^{12}$ ) fréquentes dans la poésie russe des $\mathrm{XIX}^{\mathrm{e}}$ et $\mathrm{xx}^{\mathrm{e}}$ siècles, en soulignant leur caractère "totalement incompréhensible pour un lecteur français : quel mètre voulezvous citer en français, si ce n'est l'alexandrin?» $(2015$ : 334).

Enfin, et pour envisager un cas limite, on se rend compte que le jeu avec l'intertextualité sert parfois subtilement l'imagination créatrice d'un traducteur à des fins politiques. Ainsi, dans sa traduction du Faust de Goethe, Boris Pasternak traduit-il un passage relatif à Méphistophélès en reprenant volontairement les mêmes mots qu'utilise le Diable dans Les Frères Karamazov. On peut imaginer que, dans l'hypothèse (certes utopique) où l'on (re)traduirait cette traduction de Pasternak, il faudrait rendre justice à un tel feuilletage énonciatif, si fortement signifiant dans l'univers du poète.

L'intertextualité sollicite donc l'imaginaire créatif du traducteur afin de trouver des solutions adéquates pour faire entendre toutes ces voix qui « habitent » potentiellement le texte (voire en ajouter de nouvelles ${ }^{13}$ ). Mais que penser lorsqu'il décide sciemment de supprimer un élément du millefeuille original? C'est le cas de Guerne traduisant les Sonnets à Orphée de R. M. Rilke. Ayant une aversion profonde pour Paul Valéry dont l'influence sur les Sonnets rilkéens est évidente, et au nom d'une vision toute personnelle, Guerne décide de "purifier» les poèmes allemands de leur empreinte valéryenne. Il mentionnera fièrement avoir remis aux éditions du Seuil le manuscrit français des Sonnets "aridement dévaléryanisés du Rilke envaléryanisé » (2000: 200). La créativité du traducteur apparaît donc ici assez problématique. En effet, la représentation subjective que peut avoir le poète traducteur sur l'auteur qu'il traduit ${ }^{14}$ ne s'exerce-t-elle pas au détriment de l'œuvre elle-même? L'imaginaire créatif de l'écrivain traducteur est bien susceptible, dans certains cas, de complètement transformer la lecture du texte premier. En vertu de sa position personnelle et d'un accès que l'on peut qualifier de privilégié à l'imaginaire poétique, le poète traducteur ne va-t-il pas parfois trop loin ? Vaste question, mais pour un tout autre débat. 


\section{BIBLIOGRAPHIE}

Albert-Birot, Pierre, 1945, Les Amusements naturels, Paris, Denoël.

Albrecht, Jörn, 1998, Die literarische Übersetzung, Darmstadt, Wissenschaftliche Buchgesellschaft.

Bellos, David, 2012, Le Poisson et le Bananier. Une histoire fabuleuse de la traduction, Paris,

Flammarion.

Bonnefoy, Yves, 2000, "La traduction des sonnets de Shakespeare ", dans P. Dorval et J.-

M. Maguin (dir.), Shakespeare et la France, Actes du congrès de 2000 de la Société française Shakespeare, Actes des congrès de la Société française Shakespeare, $\mathrm{n}^{\circ}$ 18, [En ligne], http://

journals.openedition.org/shakespeare/540.

DOI : $10.4000 /$ shakespeare. 540

Bonnefoy, Yves, 2013a, «Shakespeare et la poésie », dans L’Amitié et la Réflexion, Tours, Presses universitaires François-Rabelais, p. 61-75, [En ligne], https://books.openedition.org/pufr/771? lang=fr.

Bonnefoy, Yves, 2013b, L'Autre Langue à portée de voix, Paris, Seuil.

Brodsky, Joseph, 2013, Vingt sonnets à Marie Stuart, Le Mans, Les doigts dans la prose, trad. anglaise de Peter France et l'auteur, trad. française de Claude Ernoult, trad. française d'André Markowicz.

Darwich, Mahmoud, 2006, Entretiens sur la poésie, Arles, Actes Sud.

Guerne, Armel, 1975, « Novalis ou la vocation d'éternité », avant-propos, dans Novalis, Euvres complètes, vol. 1, Paris, Gallimard.

Guerne, Armel, 2000, Lettres à E. M. Cioran, Lectoure, Le Capucin.

Leyris, Pierre, « Quand T. S. Eliot parle Perse », Palimpsestes, $n^{\circ}$ 2, « Traduire la poésie », p. 19-27, [En ligne], https://journals.openedition.org/palimpsestes/719

DOI : 10.4000/palimpsestes.719

Lombez, Christine, 2004, "Traduction de la poésie en vers ou en prose ? Le cas de G. de Nerval », Transfert, $\mathrm{n}^{\circ}$ 1, « Poésie, traduction, retraduction », CNRS, Université Montpellier III.

Lombez, Christine, 2016, La Seconde Profondeur. La traduction poétique et les poètes traducteurs en Europe au $\mathrm{XX}^{e}$ siècle, Paris, Les Belles-Lettres.

Markowicz, André, 2015, Partages : un an de chroniques sur Facebook (juin 2013-juillet 2014), Paris, Inculte, Dernière Marge.

Meschonnic, Henri, 1972, «Propositions pour une poétique de la traduction », Langages, $\mathrm{n}^{\circ} 28$, p. 49-54.

Meschonnic, Henri, 1973, Pour la poétique II, Paris, Gallimard.

Robin, Armand, 1992, Fragments, Paris, Gallimard.

Sanmann, Angela, 2013, Poetische Interaktion : Französisch-deutsche Lyrikübersetzung bei F. Kemp,

P. Celan, L. Harig, V. Braun, Berlin, De Gruyter.

Sereni, Vittorio, 2013, Poesie e prose, Milan, Mondadori. 


\section{NOTES}

1. Pour parler de traduction, Cicéron par exemple avait recours à des verbes ou des périphrases tels que " convertere ", " reddere ", " latine exprimere ", etc. Il a fallu attendre le $x v^{e}$ siècle pour que traducere revête son sens linguistique actuel (à la faveur d'ailleurs d'une erreur de traduction).

2. On peut également mentionner le soleil, féminin en japonais, qui explique le fait que l'Empereur puisse être conçu comme le fils de la déesse du soleil Amaterasu, assimilé au Japon à un principe féminin.

3. Sur cette entreprise voir Lombez (2016:55-76).

4. Nous soulignons.

5. Guerne (2000: 239).

6. Cf. ici la préface de l'édition de 1994 ("Traduire les sonnets de Shakespeare ») et celle qui figure dans l'édition Gallimard de 2007 et qui contient, outre les sonnets, Vénus et Adonis et Le Viol de Lucrèce («Les Sonnets de Shakespeare et la pensée de la poésie »). Voir également Bonnefoy (2000).

7. Pour plus de détails, on se reportera ici encore à Lombez (2016:97-125).

8. Cf. le titre de l'article de Leyris, « Quand T. S. Eliot parle Perse » (1990).

9. Cf. Y. Bonnefoy (2013: 285).

10. Le poème «Green » de Verlaine, extrait de Romances sans paroles (1873) commence ainsi : «Voici des fruits, des fleurs, des feuilles et des branches".

11. Le vers de Goethe original «Wie herrlich leuchtet/Mir die Natur!» devient chez Braun «Wie herrlich leuchtet mir die Montur !».

12. Markowicz (2015: 175-178) signale par exemple le cas du poète Nikolaï Zabolotski qui utilise dans un poème de 1929 intitulé "Les démons " le mètre utilisé par Pouchkine dans son poème homonyme un siècle auparavant, expliquant qu'un tel hommage est intraduisible en français où aucun poète moderne ne pourrait citer pour le mètre un poème romantique.

13. On citera le cas de G. de Nerval qui, traduisant en 1830 le poème de Goethe «Le Roi de Thulé ", y introduisit des réminiscences de la poésie de Ronsard, appelée à son esprit par association d'idées, volontaire ou non (cf. Lombez 2004). Ou encore celui de Markowicz qui, dans sa traduction de Vingt sonnets à Marie Stuart de Brodsky, rend l'expression « ЗА ВРЕМЕНИ ГОДА » par « saisons scardanelliennes ", introduisant par là même une allusion intertextuelle forte aux poèmes de la folie de Hölderlin (qui les signait du nom de Scardanelli). Cf. Brodsky (2013:41).

14. Ainsi, pour le poète traducteur italien Sereni, « on ne traduit plus, simplement, un texte, on traduit plutôt l'écho, la répercussion que ce texte a eu en nous » (2013:327, notre traduction).

\section{RÉSUMÉS}

Avec qui le traducteur de poésie traduit-il ? Dans quelle mesure est-il accompagné dans sa tâche par d'autres voix, d'autres imaginaires que les siens, mais qui vont donner à sa traduction tout son sens? Ce dialogisme est d'autant plus intéressant à questionner qu'il conditionne (souvent à son insu) les choix interprétatifs du traducteur, surtout quand celui-ci est poète lui-même. On se demandera en quoi une traduction ainsi produite peut se lire comme un feuilleté d'imaginaires 
poétiques et quel rapport (empathique ou antagoniste) ces derniers entretiennent avec l'original étranger.

With whom does the poetry translator translate? To what extent is he accompanied by other voices, other imaginaries than his own, that will give his translation its full meaning? These questions are particularly important when we consider the interpretative choices of the translator, who might also be a poet himself. This paper probes the different poetic imaginaries and addresses the relations (empathic or antagonistic) that poetry translators develop with the source text.

\section{INDEX}

Mots-clés : traduction, poésie, imaginaires, poètes traducteurs, intertextualité

Keywords : translation, poetry, imaginaries, poets translators, intertextuality

\section{AUTEUR}

\section{CHRISTINE LOMBEZ}

Université de Nantes, L'AMo, IUF 